\title{
Three-dimensional computed tomography imaging in an animal model of emphysema
}

\author{
A.R. Froese*, K. Ask*, R. Labiris” ${ }^{\#}$ T. Farncombe ", D. Warburton ${ }^{+}$, M.D. Inman”, \\ J. Gauldie* and M. Kolb*,\#
}

ABSTRACT: Emphysema is a major health problem and novel drugs are needed. Animal disease models are pivotal in their development, but the validity and sensitivity of current tools for the evaluation of drug efficacy is limited. The usefulness of micro computed tomography (CT) as an innovative tool to assess emphysema in a mouse model was investigated.

Serial CT scans were performed in bi-weekly intervals in Smad3 knockout (KO) mice, which spontaneously develop airspace enlargement. Lung density was quantified in two- and threedimensional images and correlated to mean linear intercept and lung compliance.

CT scans of Smad $3 \mathrm{KO}$ lungs revealed a significant decrease in lung density at age 8 weeks and a further progression at age 14 weeks with respect to age-matched wild-type (WT) animals. Emphysema could be reliably assessed with both the two- and three-dimensional approach, but the three-dimensional approach was superior, due to normalisation to lung volumes and less variability. Lung compliance by week 14 was $0.053 \pm 0.005$ and $0.034 \pm 0.002 \%$ of maximum volume $\cdot \mathrm{cmH}_{2} \mathrm{O}^{-1}$ for $\mathrm{KO}$ and $\mathrm{WT}$ mice, respectively, reflecting significant physiologically relevant emphysema.

Small animal computed tomography imaging and density quantification in a reconstructed three-dimensional image is a useful tool for quantifying emphysematous changes in an animal disease model. It adds significant information to conventional assessment.

\section{KEYWORDS: Animal model, computed tomography, emphysema, noninvasive assessment}

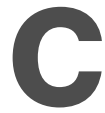
hronic obstructive pulmonary disease (COPD) and emphysema are recognised as major global health problems. The defining feature of COPD is irreversible airflow obstruction, caused by elevated resistance in the small airways and increased lung compliance due to emphysema and lung destruction [1, 2]. The pathogenesis of COPD involves repeated and/or persistent injury to the lung caused by noxious particles and gases, which lead to chronic inflammation with oxidative stress and high protease activity [3,4]. The exact mechanism of progression from tissue injury to irreversible damage in the airways and parenchyma is not completely understood [5, 6]. One prevailing hypothesis for the development of emphysema involves a protease/anti-protease imbalance, favouring a "matrix degrading micro-environment" in the lungs [7].

Animal models have substantially broadened knowledge of the development of emphysema and are instrumental for the identification of novel treatment targets [8]. The most common approach to an experimental model of emphysema is the intratracheal instillation of elastase, which causes rapid and progressive emphysema. Cigarette smoke exposure also leads to emphysema in animals but requires relatively long times to develop. There are a number of genetically altered animals, such as mice null for the Smad3 gene, which lack an integral signalling molecule in the transforming growth factor (TGF)- $\beta$ pathway and develop age-related enlargement of the alveolar airspace [9].

Airspace enlargement develops slowly and in a relatively more homogeneous manner in the Smad3 knockout (KO) mice with absence of emphysematous bullae (fig. 1), while it is patchier in the smoking model and even more so in the elastase model. Due to feasibility, drug efficacy experiments are most often performed in the elastase model [8]. For this purpose emphysema is usually evaluated by morphometry, e.g. measuring mean linear intercept (MLI), or by quantifying matrix gene expression and protein concentration. This approach seems to be fairly simple and fast, and can provide proof of principle if applied appropriately. However, morphometric techniques are not always used correctly and can be misinterpreted. FEHRENBACH [10] recently reviewed
AFFILIATIONS

*Dept of Pathology and Molecular

Medicine, Center for Gene

Therapeutics,

\# Dept of Medicine, Firestone

Institute for Respiratory Health, and

'Dept of Nuclear Medicine, Hamilton

Health Sciences, McMaster

University, Hamilton, ON, Canada.

+Developmental Biology Program,

Saban Research Institute, Children's

Hospital Los Angeles, University of

Southern California, Los Angeles, CA, USA.

CORRESPONDENCE

M. Kolb

Depts of Medicine

Pathology and Molecular Medicine McMaster University

Firestone Institute for Respiratory Health

50 Charlton Ave East

Room T2121

Hamilton L8N 4A6

ON Canada

Fax: 19055216183

E-mail: kolbm@mcmaster.ca

Received:

January 022007

Accepted after revision:

August 152007

SUPPORT STATEMENT

The present study was funded by grant support through the Canadian Institute for Health Research.

R. Labiris is a recipient of the Father Sean 0'Sullivan Career Award.

M. Kolb is supported by a career award from the Dept of Medicine at McMaster University.

STATEMENT OF INTEREST

None declared.

European Respiratory Journal

Print ISSN 0903-1936

Online ISSN 1399-3003 
the literature and noted that careful inspection of many of the animal models revealed that strict criteria of emphysema definition were demonstrated in surprisingly few of them. Furthermore, the applicability of the findings to human disease, which requires decades to develop, remains questionable. The current assessment tools cannot be repeated to follow a single animal, and may display artefacts of fixation and inflation not relevant to actual lung architecture or function.

The progressive destruction of alveoli in emphysema leads to a loss of gas exchange surface resulting in a decrease of lung parenchyma density. This structural change can be quantified in the human by a computed tomography (CT) X-ray scanner $[11,12]$. Although pulmonary function tests are the conventional method for diagnosis and clinical management of COPD and emphysema, they have weaknesses [13, 14]. CT imaging can give useful information for the diagnosis of emphysema in humans today, which may even be superior to functional
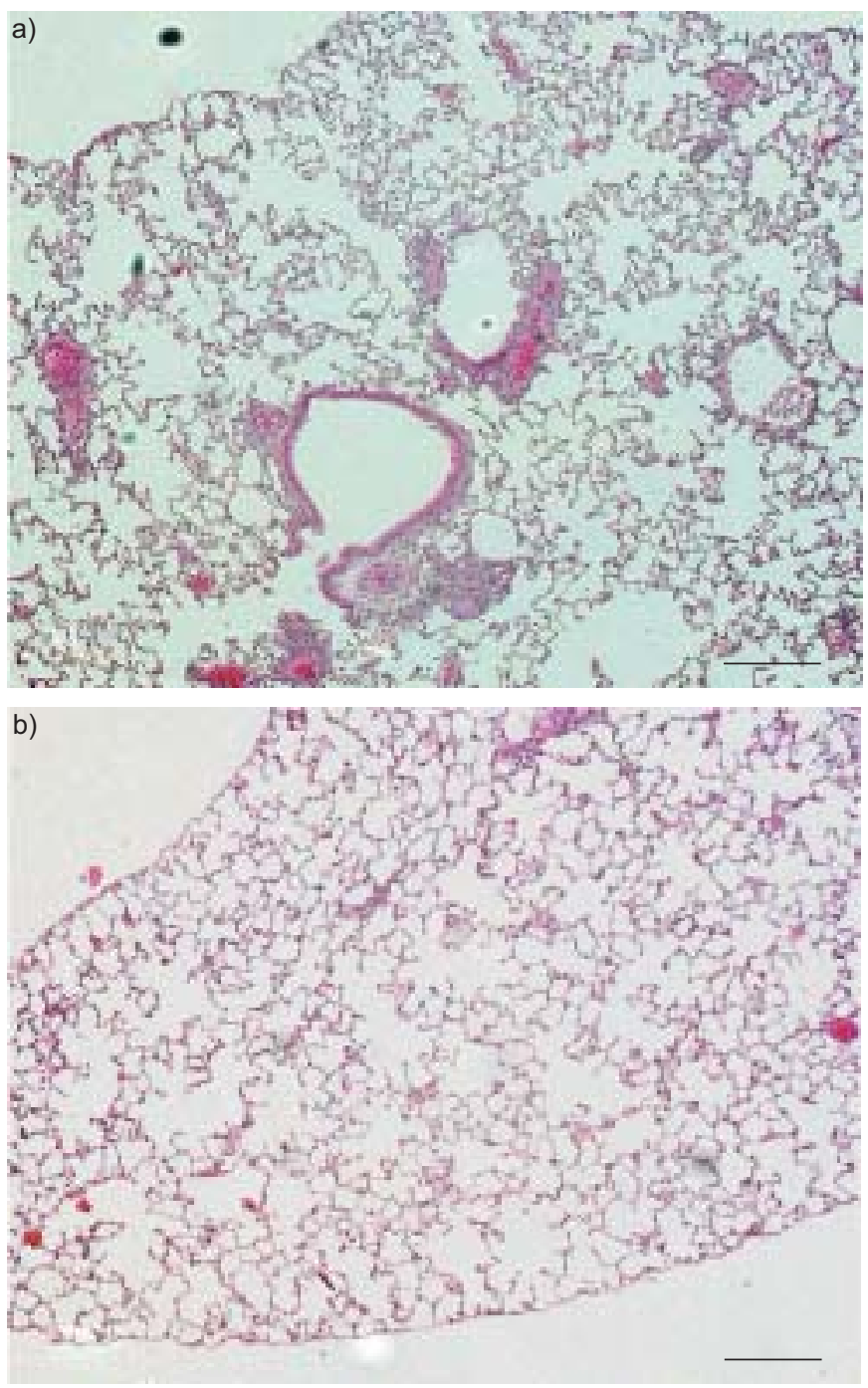

FIGURE 1. Smad3 null mice, a model of airspace enlargement. Smad3 knockout (KO) mice develop emphysema spontaneously with age. Representative histology pictures of a) a $\mathrm{KO}$ and b) a wild-type mouse at age 14 weeks. Scale bars $=100 \mu \mathrm{m}$ assessment [12]. The value of quantitative CT seems to be even stronger in assessing small changes in the lung parenchyma of human patients and may allow the visualisation of the progression of disease or quantification of changes due to interventions $[15,16]$. Micro-CT may have similar potential in assessing emphysematous changes in animal models of disease.

In the present study, micro-CT scans are validated as a new method for evaluating disease progression in an animal model of emphysema. It is shown that micro-CT correlates with both MLI in two dimensions and lung function in Smad3 KO mice $[17,18]$. The study is the first to validate two- and threedimensional micro-CT imaging in a mouse model of progressive airspace enlargement.

\section{METHODS}

\section{Animal care}

All mice were treated in accordance with the guidelines of the Canadian Council of Animal Care. All animal procedures were performed under inhalation anaesthesia with isofluorane (MTC Pharmaceuticals, Cambridge, ON, Canada) or Avertin (Aldrich Chemical Co., Milwaukee, WI, USA) without supplemental oxygen. Exon 8 of the Smad3 gene was disrupted in mice with background $129 \mathrm{SV} / \mathrm{EV} \times \mathrm{C} 57 \mathrm{BL} / 6$ [18]. Mice were bred in specific pathogen-free rooms.

\section{Histology}

For histological examination, lungs were inflated and fixed by intratracheal instillation of $10 \%$ neutral buffered formalin at a constant pressure of $20 \mathrm{cmH}_{2} \mathrm{O}$ for $5 \mathrm{~min}$. After fixation in $10 \%$ buffered formalin for $24 \mathrm{~h}$, lungs were paraffin embedded, cut in $3-\mu \mathrm{m}$ sections and stained with haematoxylin and eosin.

\section{CT scanning}

Spontaneously breathing mice were imaged under isoflurane anaesthesia using the CT portion of an X-SPECT SPECT/CT (Gamma Medica-Ideas, Northridge, CA, USA). The X-ray tube was set to $80 \mathrm{kVp}$ and $220 \mu \mathrm{A}$. Image acquisition was performed using a continuous rotation, acquiring 512 projection measurements into a $1,024 \times 1,024$ array (pixel size $0.10 \mathrm{~mm}$ ). Images were reconstructed using a Feldkamp cone beam reconstruction algorithm into $512 \times 512 \times 512$ arrays $\left(0.1157 \mathrm{~mm}^{3}\right.$ voxels $)$, and then rescaled to provide CT numbers. Whole-body X-ray dosimetry was measured using TLD 100 chips (Harshaw-Thermo RMP, Solen, OH, USA) embedded in six places in the body of dead mice and exposures were averaged. The chips were read by Global Dosimetry Solution Inc. (Irvine, CA, USA). Mice scanned for $120 \mathrm{~s}$ yielded an average whole body exposure of $\sim 27 \mathrm{mGy}$. Reconstructed image data was then analysed using Amira ${ }^{\circledR}$ software (Mercury Computer Systems, Chelmsford, MA, USA) to quantify lung tissue density. Three-dimensional volumes were drawn manually around the anatomical lungs in each image. Lung tissue density within these volumes was determined by constructing a histogram over the CT number range $-1,000--100$ using 18 bins of width 50 Hounsfield Units (HU). CT numbers were expressed as averages of the percentage of total lung volume for a given group.

Validation experiments were performed to demonstrate that the present imaging approach was able to reliably detect 
changes in densities of lung inflated with different volumes of air. Naïve Balb/c mice $(n=5)$ underwent the imaging protocol in four different conditions: spontaneously breathing under isofluorane anaesthesia, dead with open trachea, dead with trachea blocked and lungs inflated with $500 \mu \mathrm{L}$ and $700 \mu \mathrm{L}$ air.

\section{Two-dimensional morphometry to two-dimensional CT density correlation}

Transaxial histological sections from eight animals (four $\mathrm{KO}$ and four wild-type (WT)) were highlighted in select areas of airspace enlargement and normal lung tissue. MLI measurements were taken manually by counting the intercepts with 10 randomly placed lines on each of at least seven photographs per highlighted area. The closest two-dimensional transaxial CT slice was highlighted in the same selected areas and analysed for voxel count and density. The voxels with density -550- $-450 \mathrm{HU}$ were summed and analysed against the same area's MLI. These densities were selected because they correspond to normal (-450 HU) and enlarged airspaces (-550 HU).

\section{Pressure-volume loop determination}

All pressure-volume loops were performed with a quantitative mechanical respirator (flexiVent; SCIREQ Scientific Respiratory Equipment, Montreal, QC, Canada). In order to avoid possible bronchodilatory effects of isofluorane, animals were anaesthetised with Avertin (Aldrich Chemical Co.) at $240 \mathrm{mg} \cdot \mathrm{kg}^{-1}$ i.p. and paralysed with pancuronium bromide (Sandoz Canada Inc., Boucherville, QC, Canada) $0.03 \mathrm{mg} \cdot \mathrm{kg}^{-1}$ i.v. An intratracheal tube was then inserted and the animal placed on mechanical ventilation. The positive end-expiratory pressure was constant at $2 \mathrm{cmH}_{2} \mathrm{O}$.

\section{Statistical analysis}

Data are shown as mean \pm SEM or mean \pm SD. For evaluation of differences between Smad3 $\mathrm{KO}$ and WT group values, unpaired t-tests were used. ANOVA (F-test) was used in evaluating the variability between individual analysers during the inflated mouse CT validation. A value of $\mathrm{p}<0.05$ was considered significant. Linear regression was used for the comparison of two variable sets.

\section{RESULTS}

\section{Smad3 KO emphysematous progression}

The current authors have previously described the progression of airspace enlargement in Smad3 mice to be spontaneously acquired with age [9]. Figure 1 illustrates the extent of airspace enlargement seen in $\mathrm{KO}$ animals up to 14 weeks of age, whereas WT animals are essentially unchanged. Enlargements in MLI for KO animals are significant at 8 weeks, and increase further at 14 weeks of age.

\section{Two-dimensional morphometric correlation to CT density}

The histomorphology from animals with and without airspace enlargement were correlated with the same two-dimensional slice from concurrent CT scans. Areas with obvious emphysema and normal lung parenchyma were photographed and analysed for MLI (figs 2a-1). The same area in the twodimensional transaxial slice was analysed for the amount of lower density voxels at $-550--450 \mathrm{HU}$, as only these selected voxels showed correlation with MLI data. Figure 2 shows, for example, that area A1 has significantly more airspace enlargement and lower CT densities than area B1. Furthermore, the increasing low density seen in the CT scan correlated with severity of airspace enlargement seen in the histology. There was significant visible correlation between areas with normal and low density in the two-dimensional CT slice and the position of normal and emphysematous regions in the histology. Areas that contained visible airspace enlargement in the histology correlated with scan densities of $\sim-550 \mathrm{HU}$ and below. Figure $2 \mathrm{~m}$ shows the correlation between two-dimensional areas in the CT image summed through $-550--450 \mathrm{HU}$ and the same areas' MLI for 16 areas $\left(\mathrm{R}^{2}=0.53\right)$.

\section{Three-dimensional CT density analysis}

Conventional research of emphysema in animal models relies on mean chordal length measurements taken from random areas of the lung and then averaged. This analysis is usually performed on a single section of lung, thereby missing potential patchy areas of airspace enlargement. Similar limitations may apply to two-dimensional CT imaging. In contrast, three-dimensional CT images can be used to find the spatial orientation and density of mouse lungs in real time. Spatial orientation of low-density voxels may vary between subjects, but the amount of low-density voxels can be averaged from three-dimensional images to find significance between groups. Voxels with density of $-550--1,000 \mathrm{HU}$ were considered to represent emphysematous changes, since these densities were not seen in the normal lungs of WT animals. Figure 3 shows Smad3 KO and WT mice that have been serial scanned from 614 weeks of age. As the mice progress in age, a shift toward less dense tissue can be visualised. The Smad3 mouse is fairly heterogeneous, with some $\mathrm{KO}$ animals displaying more lowdensity tissue than others. Smad3 KO mice do not all progress to emphysema at the same age but, in general, increases in MLI and low-density voxels were observed at the onset of sexual maturation (6-7 weeks). Images were reconstructed in three dimensions to help visualise changes in MLI in the lungs of KO animals over time. Figure 4 shows images from one representative $\mathrm{KO}$ and one WT mouse, in which amounts of low-density tissue $(-700--550 \mathrm{HU})$, coloured yellow, increase greatly from 6 to 14 weeks. The finding that two-dimensional transaxial slices correlated well with three-dimensional densities is supported by the even distribution of airspace enlargement throughout the Smad3 KO lung.

\section{Comparison of two- and three-dimensional CT data}

In the past, two-dimensional slices were analysed to determine differences between groups. The radiologist was responsible for finding the best representative slice for the whole lung. To ascertain whether the amount of low-density tissue seen in Smad3 KO was also found in WT animals, two-dimensional slices were extracted from the top and bottom of each CT scan. As expected, the data revealed differences in the amount of low-density voxels in the range $-700--550 \mathrm{HU}$. Figure 5a shows the percentage of whole two-dimensional slices with densities -700- -550 HU. With age, the KO two-dimensional slice voxels decrease in density and are significant at 10 and 14 weeks of age with respect to WT age-matched animals $(\mathrm{p}<0.01$ and $\mathrm{p}<0.05$, respectively). On average, the density of $\mathrm{KO}$ lungs was decreased at 14 weeks with respect to $\mathrm{KO}$ animals at 6 weeks of age $(p<0.04)$. On comparing data 

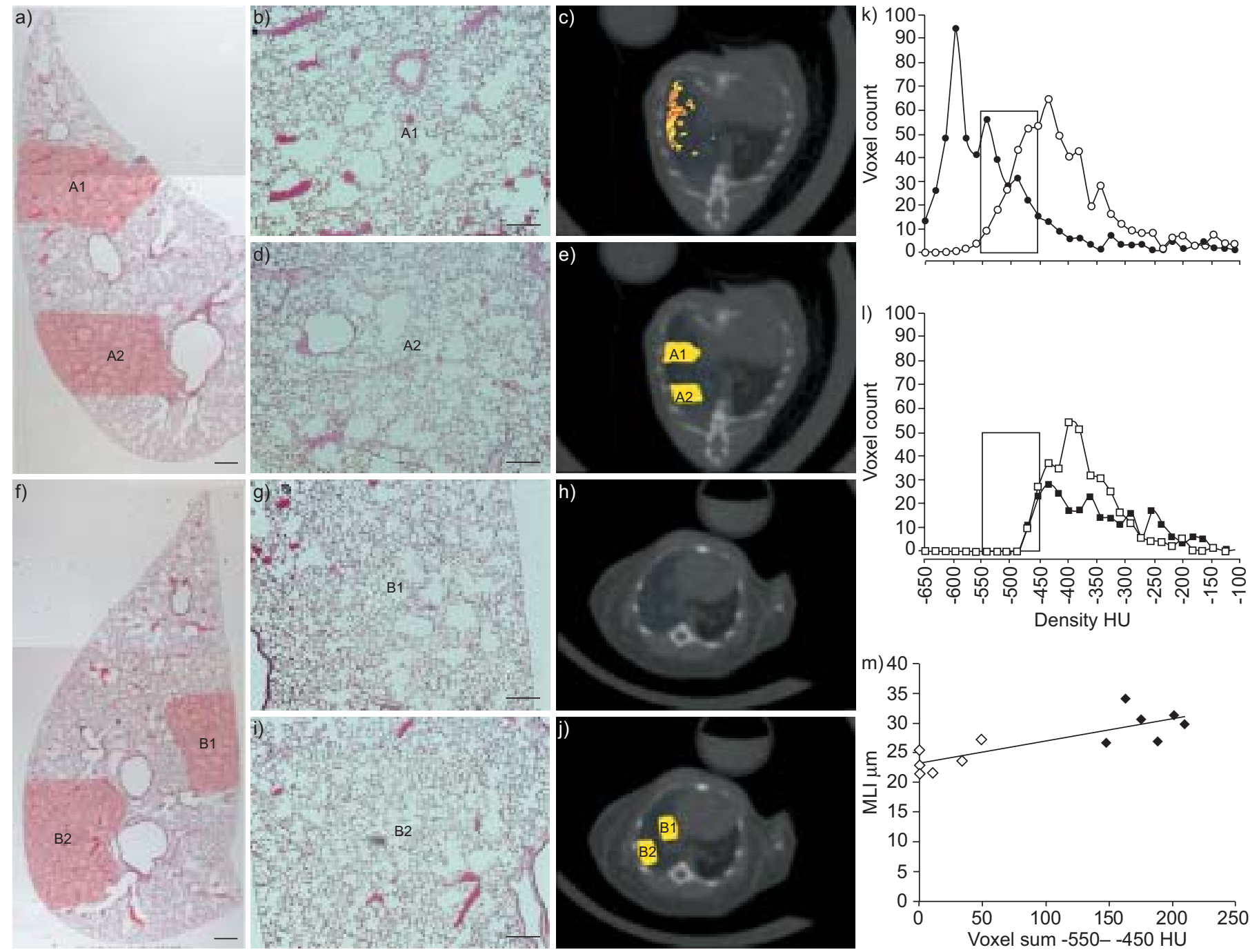

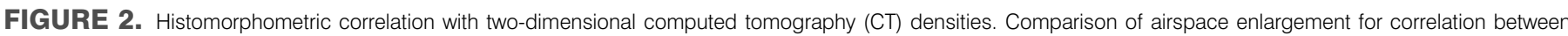

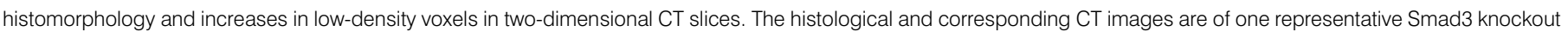

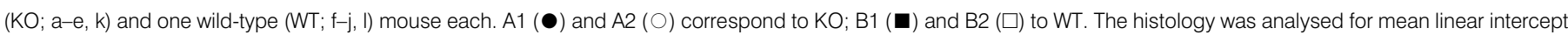

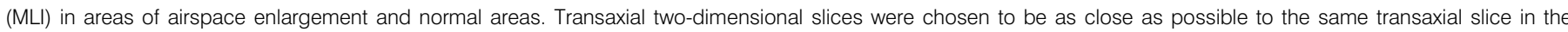

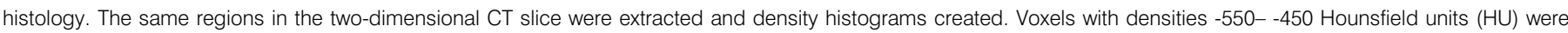

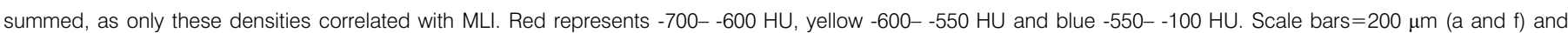

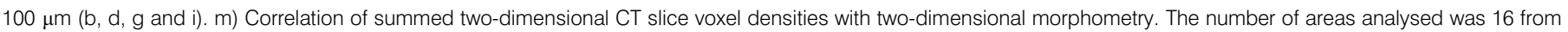
eight animals (four KO $(\diamond)$ and four WT $(\diamond)$ ). All mice analysed were 14 weeks of age. Correlation of morphology to CT densities had $\mathrm{R}^{2}=0.53$.

extracted from the two-dimensional slices to three-dimensional whole lungs, high correlation was found between the two $\left(R^{2}=0.94\right)$, suggesting that the densities from two averaged two-dimensional slices were representative of densities in the whole scan in this model. However, despite the homogeneous pattern of airspace enlargement in Smad3 KO mice, threedimensional data had less variability $(\mathrm{CV}=1.43)$ compared with two-dimensional variability $(\mathrm{CV}=1.24)$. The difference in summed low-density voxels of $\mathrm{KO}$ lung in comparison with controls achieved higher levels of significance in threedimensional analysis compared with two-dimensional analysis $(p=0.00007$ versus $p=0.0005)$. It can be anticipated that the variability would be markedly increased in a model with patchier tissue changes (fig. $5 b$ and $5 c$ ).

\section{Validation of lung density analysis}

Validation experiments were performed to demonstrate the validity of the present imaging method in detection of changes in lung densities. Naïve Balb/c mice $(n=5)$ underwent the imaging protocol in four different conditions: spontaneously breathing, dead with open trachea, dead with trachea blocked, and lungs inflated with $500 \mu \mathrm{L}$ and $700 \mu \mathrm{L}$ air. The mean lung volume of spontaneous breathing mice weighing 20-30 g was $619 \pm 63.4 \mu \mathrm{L}$ with a maximum voxel count at densities -425 $-375 \mathrm{HU}$. After euthanasia, the mean lung volume was $352 \pm 20.2 \mu \mathrm{L}$ with a maximum voxel count at densities $-375--325$ HU. With inflation of the lung with known air volumes, the change in lung volume calculated from the CT scan was $485 \pm 47 \mu \mathrm{L}$ after inflation with $500 \mu \mathrm{L}$ and 

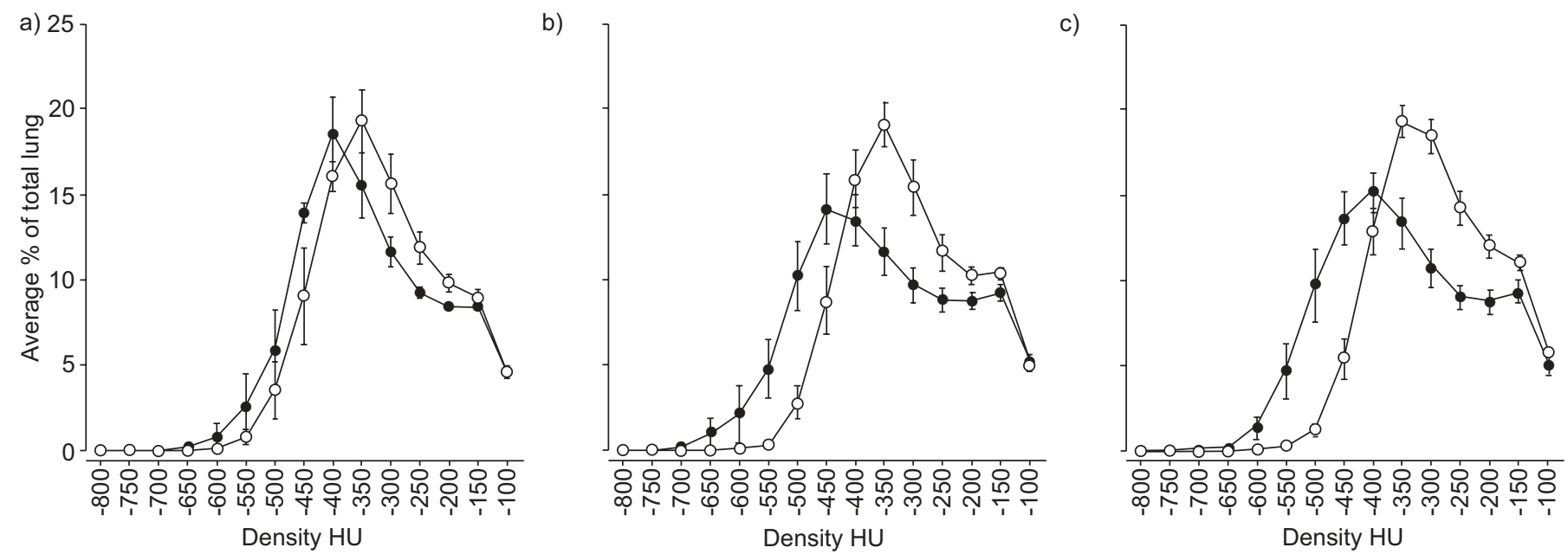

FIGURE 3. Progression of Smad3 knockout (KO) and wild-type (WT) mice airspace enlargement with age. Average percentage of total lung volume at time of scan at a) 6 weeks $(n=4 \mathrm{KO}, n=6$ WT); b) 10 weeks $(n=8 \mathrm{KO}, \mathrm{n}=11 \mathrm{WT})$; and c) 14 weeks $(n=9 \mathrm{KO}, \mathrm{n}=15 \mathrm{WT})$. $\bullet: \mathrm{KO}$; $\bigcirc$ : WT. Data are presented as mean \pm sEM.

$748 \pm 27 \mu \mathrm{L}$ after $700 \mu \mathrm{L}$ of air (fig. 6). Note that traditional human emphysematous CT densities in the area of $-950 \mathrm{HU}$ are never reached by over-inflated mice. The quantitative analysis of the CT images was performed independently by three different analysers. The total lung volume was determined by each analyser and averaged for each mouse. Using

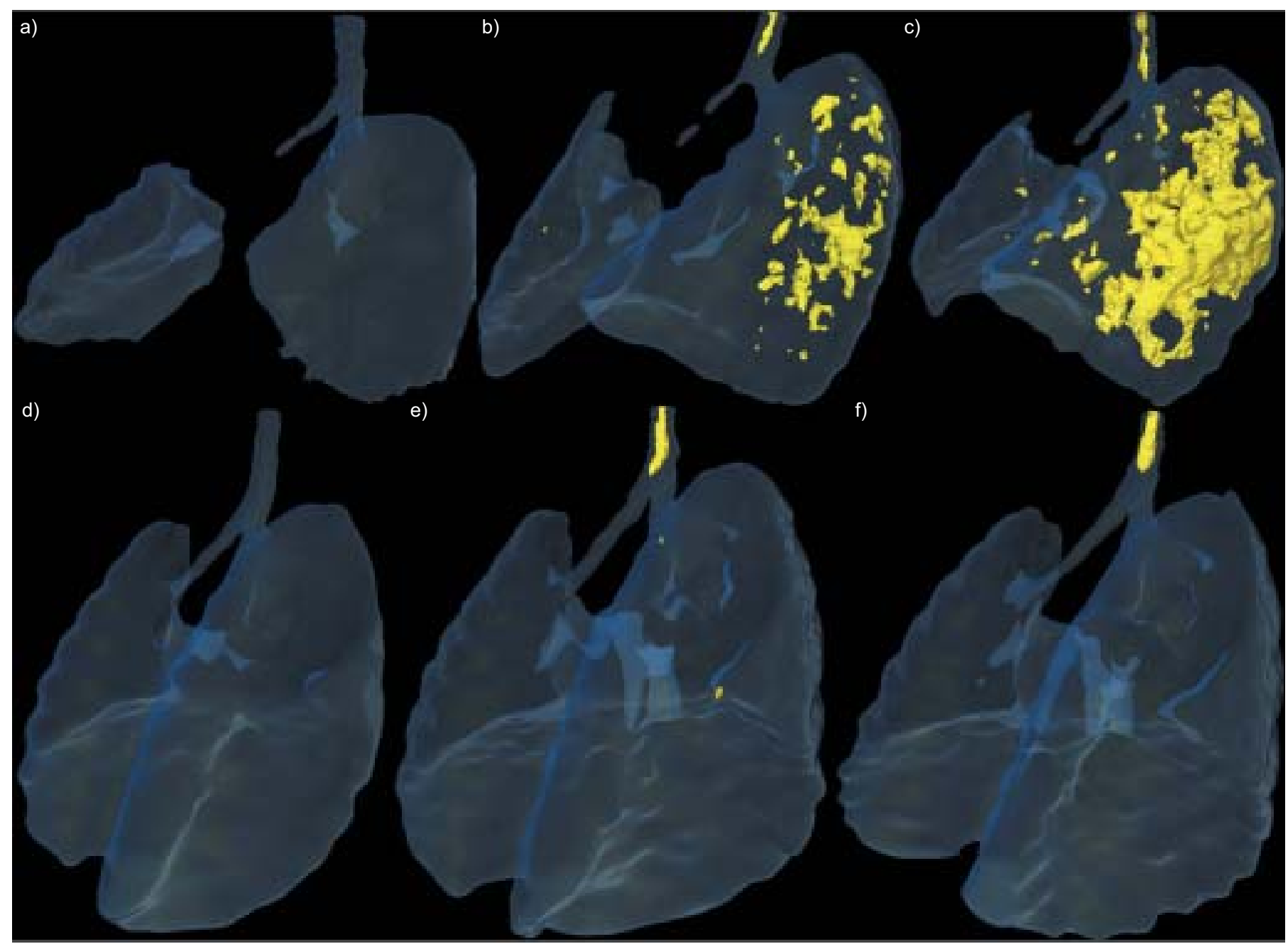

FIGURE 4. Three-dimensional images displaying decreased lung density with time in representative knockout (a-c) and wild-type (d-f) mice at age 6 (a and d), 10 (b and e) and 14 (c and f) weeks. Yellow colour represents voxels with densities - 700- $-550 \mathrm{HU}$, blue represents voxels with densities $-550--100 \mathrm{HU}$. 

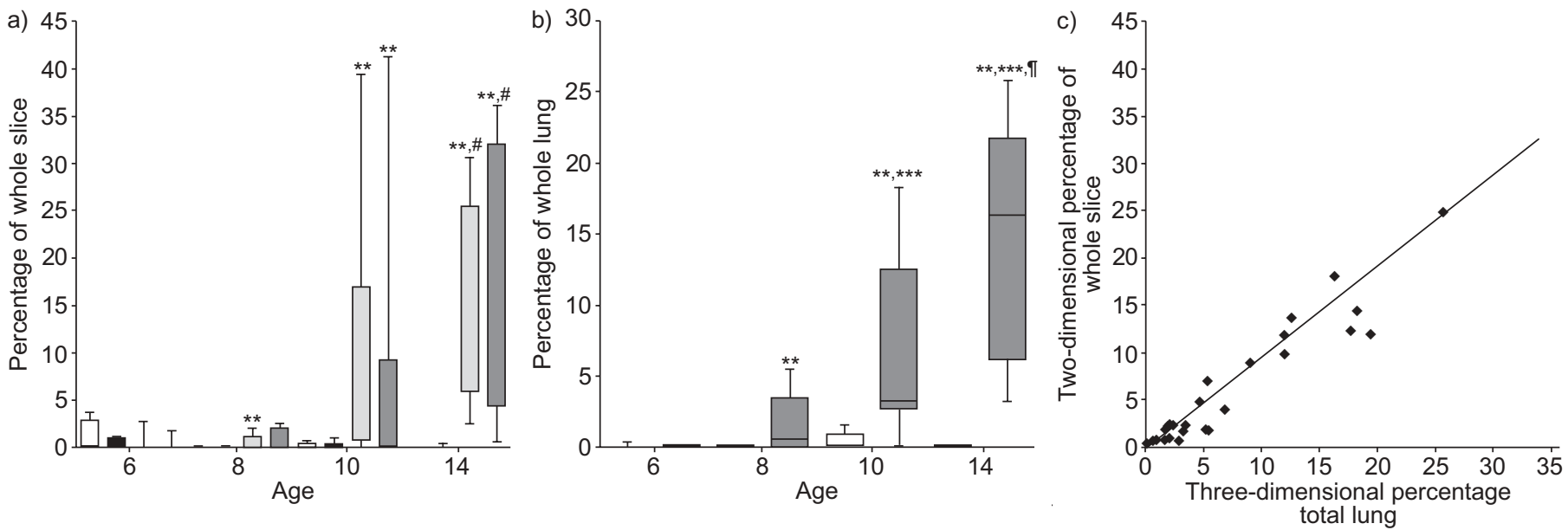

FIGURE 5. Correlation of two- and three-dimensional computed tomography densities. a) Average percentage of whole two-dimensional slices with densities -700$-550 \mathrm{HU}$. Two slices were extracted per scan. The top slice ( $\mathbf{\square}$ : wild-type (WT); $\mathbf{\square}$ : knockout (KO)) was always 32 slices under the top of the lung and the bottom slice ( $\square$ : WT; II: KO) was taken 32 slices above the bottom of the lung. b) Average percentage of whole three-dimensional lung with densities $-700--550 \mathrm{HU}$. $\square:$ WT; $\square:$ KO. Box plots are shown as mean \pm SD. C) Correlation of two- and three-dimensional density data. The percentages of total two-dimensional slices with density - $700--550 \mathrm{HU}$ (Iow density) were averaged between the top and bottom of the lung. The three-dimensional percentage of low density in the whole lung was compared against the same scan's twodimensional slice average with a correlation of $\mathrm{R}^{2}=0.94$. At 6 weeks, four $\mathrm{KO}$ and six WT observations were made; at 10 weeks, eight $\mathrm{KO}$ and $11 \mathrm{WT}$; and at 14 weeks, nine $\mathrm{KO}$ and $15 \mathrm{WT}$. ${ }^{* *}: \mathrm{p}<0.01$ with respect to age-matched WT; ${ }^{* *}$ : $p<0.001$ with respect to KO at age 6 weeks; ${ }^{\#}: \mathrm{p}<0.04$ with respect to 6 - or 8-week KO; ${ }^{*}: \mathrm{p}<0.006$ with respect to $\mathrm{KO}$ at 6 or 8 weeks.

the F-test, there was insufficient evidence to conclude that there was a difference between analysers with a $98 \%$ confidence interval. One additional experiment was performed on one mouse, which was imaged at eight different times to determine the repeatability of the measurements. The coefficient of variation of total lung volume among the eight scans was $7.7 \%$. These results confirm that calculation of lung volume from the CT image was sensitive to changes in air volume, repeatable and has a high inter-analyser reliability.

\section{Physiological correlation: pressure-volume loop}

Pressure-volume loops were measured ex vivo in order to determine whether or not the changes seen in the CT density figures actually represented physiological differences in lung compliance. Figure 7a shows the average quasi-static pressure-volume loop of $\mathrm{KO}$ and WT animals. Figure $7 \mathrm{~b}$ illustrates that at 14 weeks of age the Smad3 KO mice show a marked increase in compliance compared with the WT controls $(\mathrm{p}<0.007)$.

\section{DISCUSSION}

Animal models of emphysema are pivotal in identifying and evaluating novel therapeutic interventions in this disease. The current approaches to the assessment of drug efficacy in emphysema models have limitations, and the transferability to human disease remains questionable. The present study shows data representing the attempt to use CT imaging as a method to follow the progression of emphysema in a mouse model using two- and three-dimensional quantitative analysis over time. The advantages of this method are that it is reliable, fast and diagnostic of emphysema-like changes. Furthermore, it has the advantage of longitudinal follow-up of individual animals and avoids problems associated with fixation artefacts and morphometry.
Knowledge of the molecular mechanisms involved in emphysema development steadily increases, substantially assisted by animal disease models [8]. Intratracheal instillation of noxious hazards, such as elastase or cigarette smoke exposure, can cause emphysema. Similarly, emphysema develops spontaneously in naturally occurring mutant mouse strains or in genetically altered animals. Novel molecular targets have been identified and drugs are being developed to interfere with the molecular mechanisms of emphysema. Due to time constraints and feasibility, drug efficacy experiments are usually carried out in the elastase model [8], which provides early end-points and a rapid readout. Emphysema is currently evaluated by histomorphometry, and reliable determination of MLI or chordal length requires careful and standardised inflation of the lungs. Any leak in the airways or on the pleural surface would result in incorrect measurements [19]. The lung images shown in figures 1 and 2 were purposely obtained at low magnification to illustrate that even a validated method using standardised inflation pressure to prepare tissue slides for histomorphometry does not always result in the ideal images that are shown in textbooks.

The present study shows that CT imaging in animal models of emphysema is not only able to provide reliable quantification of airspace enlargement similar to histomorphometry but has several advantages over the conventional methods. Noninvasive CT scanning does not require euthanasia and can be performed as needed at several time points. It also can be repeated in individual animals to visualise a dynamic disease progression, or reversibility in the case of drug efficacy studies. This not only helps to account for interindividual variability but also reduces the number of animals required for a study.

The current technique was chosen due to its short image acquisition time and the fact that it requires the lowest dose of 


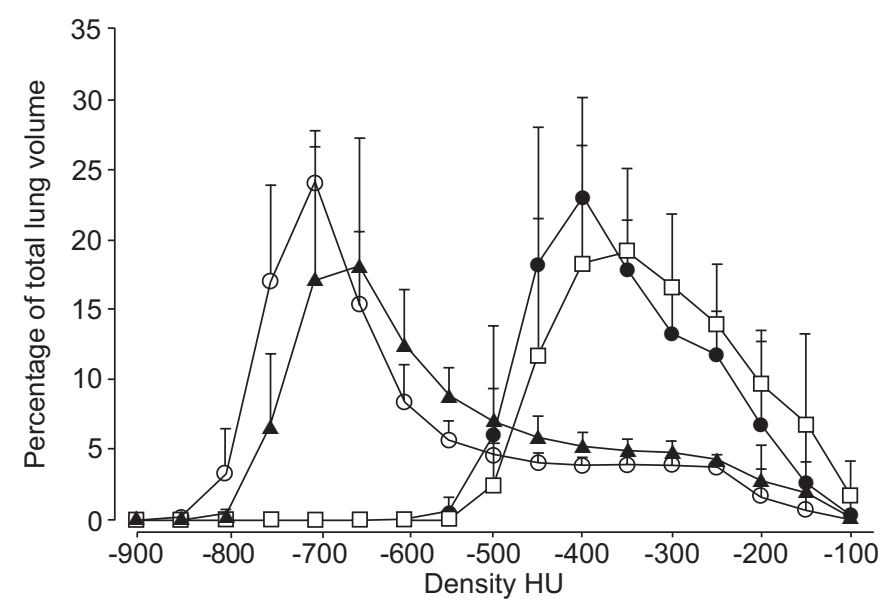

FIGURE 6. Validation of inflated lung volumes to computed tomography densities. Average percentage of total lung volumes for mice scanned alive ( $n=5)$, dead with open trachea $(\square, n=5)$, dead with trachea closed and either $500 \mu \mathrm{L}(\boldsymbol{\Lambda}, \mathrm{n}=5)$ or $700 \mu \mathrm{L}(\mathrm{O}, \mathrm{n}=3)$ of air injected. Data are shown as mean $\pm \mathrm{SD}$.

radiation for detection of emphysematous progression. Acquisition of data is swift, requiring only 2 min per scan. The data can be then reconstructed in another $2 \mathrm{~min}$ and further analysed for three-dimensional assessment using Amira ${ }_{\circledR}$ imaging software in another $5 \mathrm{~min}$. The present authors determined that the radiation dose of a 120-s scan is $\sim 27 \mathrm{mGy}$, some 50 times less than values capable of significant lung damage [20]. The resolution of the scans is $115 \mathrm{~mm}^{3}$, which, although large compared with the finest micro-CT resolutions, shows significant and meaningful differences between animals with and without emphysema. It is noteworthy that the animals in the present study were under general anaesthesia but spontaneously breathing, thus each animal was analysed at a volume close to its functional residual capacity, which may well have changed between groups, based on the observed differences in the pressurevolume loops. Had it been chosen to ventilate the animals, the problem of changing the lung volume depending on the ventilator frequency would have been introduced [21]. The current authors consider these measurements as evidence of functional emphysema in the Smad3 $\mathrm{KO}$ mice. For histological analysis, mouse lungs were inflated to $20 \mathrm{cmH}_{2} \mathrm{O}$ and formalin fixated. This was performed as a means to standardise all lungs to a fixed point on their pressure-volume relationship prior to analysing for evidence of airspace enlargement. Given that there were obviously differences in lung volumes at the time of functional and histological assessment, any direct comparisons in terms of airspace size between these conditions would not be appropriate. Using these independent methods, it was shown that Smad3 KO mice develop airspace enlargement over time, and it was demonstrated in follow-up studies of individual mice that these emphysema-like changes correlate significantly with two-dimensional morphometry. The current authors further show that the loss of lung parenchyma, as assessed by decrease in density in the threedimensional image, is associated with increased compliance of the lungs, strengthening the validity of the imaging technique as a meaningful outcome for assessment of an animal model of emphysema.
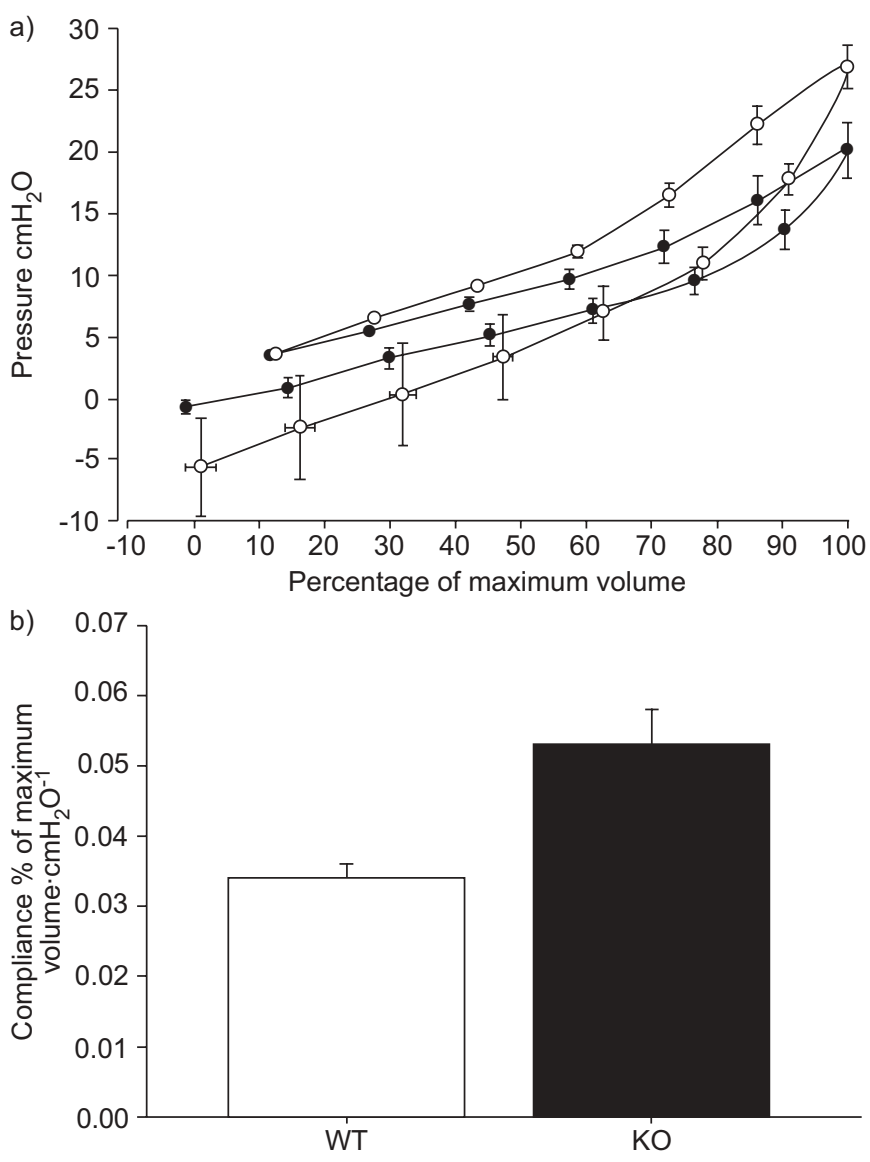

FIGURE 7. Physiological properties of Smad3 lungs. a) Average quasi-static pressure-volume loop of Smad3 knockout (KO; - ) and wild-type (WT; $\bigcirc)$ mice at age 14 weeks ( $n=8 \mathrm{KO}, \mathrm{n}=15 \mathrm{WT}$ ). b) Significant difference in lung compliance between Smad3 KO and WT mice $(p<0.007)$. Compliance was measured by the slope of the last eight points of the lower line of the average pressure-volume loop at 14 weeks of age. Data are shown as mean \pm SEM.

\section{Why a three-dimensional approach?}

The most common form of emphysema is smoking related and develops from enlarged terminal bronchioli, progressing in a centrilobular pattern. The lung apices are usually more involved than the bases. Panacinar emphysema typically is associated with $\alpha_{1}$ - antitrypsin deficiency, is more present in the bases, and overall is more uniform than the patchier distribution of the former [1]. Data from older studies suggest that one or the other of these emphysema types usually dominates in advanced disease [22], emphasising that emphysema develops in a heterogeneous manner and that single-slice histomorphometry at a single time point may not be sufficient to obtain reliable data. Furthermore, it is well known that only $\sim 15 \%$ of smokers develop clinically symptomatic COPD, whereas $40 \%$ show signs of emphysema [23], suggesting a long subclinical course of emphysema. Emphysema in animal disease models can progress in a similar patchy pattern from a centriacinar lesion, depending on the initiating agent and species of animal used in the experiment [24]. To date, most imaging-related questions asked in animal models of emphysema have relied on two-dimensional CT slices. The patchy pattern of emphysema may be lost in histomorphology or 
two-dimensional imaging, but is not lost when using a threedimensional approach [25]. The Smad3 KO model described herein seems to have slightly more airspace enlargement in the central areas of the lungs, but the decreases in density are fairly evenly present toward the top and bottom of the lung. Although two- and three-dimensional image analysis correlated significantly in the present study, a higher variability was still found in lung density assessed by two-dimensional slice analysis compared with the three-dimensional approach. It can be anticipated that the variability of two-dimensional data will be markedly higher in a model with patchier emphysema, such as the elastase model, which is commonly used for drug efficacy experiments. Furthermore, any technique involving slices of a three-dimensional structure may imply some bias on the part of the investigator during the attempt to find the most representative slices. Finally, three but not two dimensionally based analysis of lung density is adjusted to lung volume. Air trapping, a dynamic hyperinflation of the lungs but not structural emphysema would appear as an area with low density in a two-dimensional image and, as such, labelled as emphysema, whereas in three-dimensional image analysis, it would be related to lung volume and not be considered emphysema. In addition, three-dimensional image analysis is substantially faster than two-dimensional with the availability of appropriate software. All this makes three-dimensional reconstruction of the $\mathrm{CT}$ image and quantification of lung density a powerful tool that seems to be more efficient than conventional two-dimensional assessment.

\section{Conclusion}

It has been demonstrated that computed tomography imaging and density quantification in a reconstructed three-dimensional image is a useful tool for the quantification of emphysema-like changes in an animal disease model. It is at least as reliable as conventional histomorphometry but has the advantage that individual animals can be followed over time. The method is fast, easy to perform and cost efficient. The current authors believe that this assessment tool will be extremely valuable in drug efficacy experiments in emphysema research.

\section{ACKNOWLEDGEMENTS}

The authors acknowledge the expert technical assistance of R. Ellis, C. Lavery, T. Rahman, C. Saab and J. Wattie, and D. Boreham for performing radiation dosimetry (all Hamilton, ON, Canada).

\section{REFERENCES}

1 Hogg JC. Pathophysiology of airflow limitation in chronic obstructive pulmonary disease. Lancet 2004; 364: 709-721.

2 Keller CA. Pathophysiology and classification of emphysema. Chest Surg Clin N Am 2003; 13: 589-613.

3 Sethi JM, Rochester CL. Smoking and chronic obstructive pulmonary disease. Clin Chest Med 2000; 21: 67-86: viii.

4 MacNee W. Pathogenesis of chronic obstructive pulmonary disease. Proc Am Thorac Soc 2005; 2: 258-266.

5 Churg A, Wright JL. Proteases and emphysema. Curr Opin Pulm Med 2005; 11: 153-159.
6 Coakley RJ, Taggart C, O'Neill S, McElvaney NG. $\alpha_{1-}$ Antitrypsin deficiency: biological answers to clinical questions. Am J Med Sci 2001; 321: 33-41.

7 Ohnishi K, Takagi M, Kurokawa Y, Satomi S, Konttinen YT. Matrix metalloproteinase-mediated extracellular matrix protein degradation in human pulmonary emphysema. Lab Invest 1998; 78: 1077-1087.

8 Mahadeva R, Shapiro SD. Chronic obstructive pulmonary disease 3: Experimental animal models of pulmonary emphysema. Thorax 2002; 57: 908-914.

9 Bonniaud P, Kolb M, Galt T, et al. Smad3 null mice develop airspace enlargement and are resistant to TGF- $\beta$-mediated pulmonary fibrosis. J Immunol 2004; 173: 2099-2108.

10 Fehrenbach H. Animal models of pulmonary emphysema: a stereologist's perspective. Eur Respir Rev 2006; 15: 136-147.

11 Müller NL, Coxson $H$. Chronic obstructive pulmonary disease. 4: imaging the lungs in patients with chronic obstructive pulmonary disease. Thorax 2002; 57: 982-985.

12 Coxson H, Rogers R. Quantitative computed tomography of chronic obstructive pulmonary disease. Acad Radiol 2005; 12: 1457-1463.

13 Rennard SI. Chronic obstructive pulmonary disease: linking outcomes and pathobiology of disease modification. Proc Am Thorac Soc 2006; 3: 276-280.

14 Uppaluri R, Mitsa T, Sonka M, Hoffman EA, McLennan G. Quantification of pulmonary emphysema from lung computed tomography images. Am J Respir Crit Care Med 1997; 156: 248-254.

15 Stolk J, Versteegh MI, Montenij LJ, et al. Densitometry for assessment of effect of lung volume reduction surgery for emphysema. Eur Respir J 2007; 29: 1138-1143.

16 Coxson HO. Computed tomography and monitoring of emphysema. Eur Respir J 2007; 29: 1075-1077.

17 Harris R. Pressure-volume curves of the respiratory system. Respir Care 2005; 50: 78-98.

18 Yang X, Letterio JJ, Lechleider RJ, et al. Targeted disruption of SMAD3 results in impaired mucosal immunity and diminished T cell responsiveness to TGF- $\beta$. EMBO J 1999; 18: 1280-1291.

19 Parameswaran H, Majumdar A, Ito S, Alencar AM, Suki B. Quantitative characterization of airspace enlargement in emphysema. J Appl Physiol 2006; 100: 186-193.

20 Coggle JE, Lambert BE, Moores SR. Radiation effects in the lung. Environ Health Perspect 1986; 70: 261-291.

21 Volgyesi GA, Tremblay LN, Webster P, Zamel N, Slutsky AS. A new ventilator for monitoring lung mechanics in small animals. J Appl Physiol 2000; 89: 413-421.

22 Kim WD, Eidelman DH, Izquierdo JL, Ghezzo $\mathrm{H}$, Saetta MP, Cosio MG. Centrilobular and panlobular emphysema in smokers. Two distinct morphologic and functional entities. Am Rev Respir Dis 1991; 144: 1385-1390.

23 Hogg JC, Wright JL, Wiggs BR, Coxson HO, Opazo Saez A, Pare PD. Lung structure and function in cigarette smokers. Thorax 1994; 49: 473-478.

24 Wright JL, Churg A. Animal models of cigarette smokeinduced COPD. Chest 2002; 122: Suppl. 6, 301S-306S.

25 Postnov AA, Meurrens K, Weiler H, et al. In vivo assessment of emphysema in mice by high resolution X-ray microtomography. J Microsc 2005; 220: 70-75. 